\title{
QoS Routing with worst-case delay constraints: models, algorithms and performance analysis
}

\author{
Antonio Frangioni ${ }^{\mathrm{a}}$, Laura Galli ${ }^{\mathrm{a}}$, Giovanni Stea $^{\mathrm{b}}$ \\ ${ }^{a}$ Dipartimento di Informatica, Università di Pisa, Largo B. Pontecorvo 3, 56127 Pisa, Italy. E-mail: \\ frangio@di.unipi.it, laura.galli@unipi.it \\ ${ }^{b}$ Dipartimento di Ingegneria dell'Informazione, Università di Pisa, Largo Lucio Lazzarino 1, 56122 Pisa, Italy. \\ E-mail: giovanni.stea@unipi.it
}

\begin{abstract}
In a network where weighted fair-queueing schedulers are used at each link, a flow is guaranteed an end-to-end worst-case delays which depends on the rate reserved for it at each link it traverses. Therefore, it is possible to compute resource-constrained paths that meet target delay constraints, and optimize some key performance metrics (e.g., minimize the overall reserved rate, maximize the remaining capacity at bottleneck links, etc.). Despite the large amount of literature that has appeared on weighted fair-queueing schedulers since the mid '90s, this has so far been done only for a single type of scheduler, probably because the complexity of solving the problem in general appeared forbidding. In this paper, we formulate and solve the optimal path computation and resource allocation problem for a broad category of weighted fair-queueing schedulers, from those emulating a Generalized Processor Sharing fluid server to variants of Deficit Round Robin. We classify schedulers according to their latency expressions, and show that a significant divide exists between those where routing a new flow affects the performance of existing flows, and those for which this do not happen. For the former, explicit admission control constraints are required to ensure that existing flows still meet their deadline afterwards. However, despite this major difference and the differences among categories of schedulers, the problem can always be formulated as a Mixed-Integer Second-Order Cone problem (MI-SOCP), and be solved at optimality in splitsecond times even in fairly large networks.
\end{abstract}

Keywords: QoS routing, worst-case delay, weighted fair-queueing, admission control, optimization

\section{Introduction}

The research on Quality of Service (QoS) in the '90s has produced a vast number of packet scheduling algorithms, to be employed at network links to determine whose flow's head-of-line

\footnotetext{
${ }^{*}$ Corresponding author

Email address: giovanni.stea@unipi.it (Giovanni Stea)
} 
packet should be sent on the link when more than one flow is backlogged. Many of these algorithms 5 aim to approximate Generalized Processor Sharing (GPS, [1]), also called Weighted Fair Queueing (WFQ). The latter is an ideal paradigm, which emulates a fluid reference system where backlogged flows are served simultaneously, and each one is given a share of the link proportional to its weight. If the weights are chosen as the flows' requested rates, then each flow always gets no less than its requested rate as long as the sum of the rates does not exceed the link capacity.

Several packet schedulers have been proposed that approximate GPS in an environment where only one packet at a time can be transmitted, instead of several simultaneously. These strike various trade-offs between accuracy of approximation and implementation complexity, two properties that have been proved to be in opposition [2]. In particular, the accuracy of a scheduler can be measured by its latency, i.e., its worst-case scheduling delay; in other words, the maximum lag of a scheduler's service with respect to GPS. The smaller this lag, the more closely that scheduler's operation will resemble that of GPS.

At one end of the spectrum lie Packet-by-packet Generalized Processor Sharing (PGPS) and Worst-case Fair Weighted Fair Queueing $\left(\mathrm{WF}^{2} \mathrm{Q}\right)$, both exhibiting the smallest possible latency. More specifically, their latency is inversely proportional to the requested rate, plus a small additive constant; we thus call these Strictly Rate-proportional (SRP) schedulers. Their downside is that they have a relatively high complexity, i.e., $O(\log n), n$ being the number of active flow $\mathrm{I}^{1}$. This might be an issue on high-speed links, where scheduling decisions have to be made in a packet transmission time (few nanoseconds) and many flows can be active simultaneously.

At the other end of the spectrum we find instead Frame-based (FB) schedulers such as Deficit 25 Round-robin (DRR) [4 and its derivatives (e.g., [5, 6, 7]). These have constant (i.e., $O(1)$ ) complexity, a result that is only possible because the order of service of backlogged flows is constant over time. As a consequence, their latency is looser than those of the SRP schedulers, since it includes a term which grows linearly with the number of flows, corresponding to the time it takes to cycle through all the flows.

In between these two extremes, we have two other possibilities. On one hand, approximations of the GPS paradigm based on flow grouping [8, 9, which achieve $O(1)$ complexity by using clever data structures, but constraining flow rates to be integer multiples of a basic quantity. The latency expression of these Group-based (GB) schedulers is similar to that of SRP schedulers, albeit with higher multiplicative and additive constants. On the other hand, schedulers that dispense with some of the intricacies of emulating a GPS server, hence have a higher latency, still at $O(\log n)$

\footnotetext{
${ }^{1}$ Note that the complexity was believed to be $O(n)$ until [3] proved otherwise. This misconception, lasting for about a decade, made pursuing approximations of these schedulers at $O(\log n)$ complexity a worthy task, which was in fact undertaken by several researchers.
} 
complexity. This is the case, for instance, of Self-clocked Fair Queueing (SCFQ, 10]). The latency of this scheduler has an additive term that grows linearly with the number of flows, hence we call it Weakly Rate-proportional (WRP).

When WFQ packet schedulers are employed, it is possible to compute the worst-case end40 to-end delay (WCD) of a flow in a multi-hop path, once its settings - specifically, its minimum guaranteed rate and latency - at each link it traverses are given. In particular, the WCD expression includes the sum of the latencies at all traversed links. A WCD guarantee is important for many applications, e.g., playback-based ones (voice, video, ... ) and real-time ones (machine-to-machine applications, augmented/virtual reality, automated trading, ...). The above-mentioned property allows one to select rates on the given path so that the WCD stays below a pre-specified deadline. In turn, this paves the way to algorithms that compute network paths where enough rate is available to meet that deadline, something that we call delay-constrained routing (DCR) henceforth.

DCR belongs to the field of QoS routing, also well researched in the last two decades. However, most of the works on delay-based QoS routing assume static additive per-link delays (e.g., 1], hence neglect the contribution of queueing to the overall end-to-end delay. Alternatively, stochastic traffic models are used to compute average end-to-end delays (e.g., [12]), which however do not provide reliable guarantees on worst-case ones, and therefore cannot be used in sensitive applications. Two works concerned with DCR are [13, 14, which show that path computation assuming SRP schedulers is $\mathcal{N} \mathcal{P}$-hard in general. If, however, the same rate is reserved at each link, then DCR becomes a polynomial problem. As recently shown in [15, 16], reserving the same rate at each link is largely suboptimal, i.e., a significant fraction of path requests are rejected unnecessarily only because of that assumption. Furthermore, despite $\mathcal{N} \mathcal{P}$-hardness, optimal solutions can be found in split-second times even in large-scale networks.

These initial results call for a more systematical investigation of the DCR problem. In [15, 16. only SRP schedulers are treated, and no result exists for the other three categories (GB, WRP, FB), despite the fact that most of these schedulers have been known for decades and are used in practice in today's equipment (especially DRR and variants thereof). This means that the tradeoff between employing lower-complexity schedulers (e.g., GB or FB ones) and the corresponding utilization of the network has not been properly characterized yet. This work aims at filling this gap by formulating the DCR problem for all the above-mentioned categories of schedulers. More specifically, given the current state of the network, a reservation cost per unit of rate for each link, a source, a destination and a WCD deadline, we determine a path along which the new flow can be routed and the corresponding rate reservation on each link (if they exist) so that the deadline is met, while existing flows still meet theirs, at the minimum possible reservation cost. The fact that the latency of a scheduler does or does not depend on the other flows (their number and/or current reserved rates) simultaneously present on the link affects the way admission control needs 
to be done at flow setup. With SRP and GB schedulers, a new flow can always be admitted as long as the used links have enough rate; hence admitting a new flow will never jeopardize the guarantees of pre-existing flows traversing the same links. For WRP and FB schedulers, instead, checking rates is not sufficient: a flow may in fact be rejected even when there is enough rate, because admitting it would make other flows violate their delay guarantees, since their latency would grow if the new flow were admitted.

In this paper, we show that the DCR problem can always be formulated as a Mixed-Integer Second Order Cone Program (MI-SOCP), for all the above scheduler categories, through careful, non-obvious modeling choices. While MI-SOCPs are in general $\mathcal{N} \mathcal{P}$-hard, they have a convex continuous relaxation, which may make them routinely solvable by today's general-purpose solvers such as CPLEX if the number of variables is not too high. This allows us to compute optimal paths in networks of fairly large size in hundreds of milliseconds at the worst. We remark that this was not, ex-ante, obvious: convexity is a rather "fragile" property, that is easily lost even with apparently very minor modifications of the functions. Indeed, our result strictly hinges on the fact that only one flow is optimized, with the others being kept at their previous state (in terms of both path and reserved rates on the links): some - although not all —of the developed formulæ would not be jointly convex in all the problem's variables if more than one flow would be optimized simultaneously. Furthermore, the fact that the obtained MI-SOCPs can be solved this quickly hinges on at least two important facts. The first is the recent breakthrough in the efficiency of solution algorithms for Mixed Integer-Nonlinear Problems (MI-NLPs), of which MI-SOCPs are a subclass [17. The second is the use of nontrivial formulations, in particular obtained through the Perspective Reformulation technique [18, 19, which yield tighter lower bounds and therefore significantly increase the efficiency of the solution processes [15]. Only the combined effect of these 95 improvements allows us to coalesce in the same framework combinatorial constraints, such as those of routing, and non-linear ones, such as those of worst-case delays, in a solution time which would have been unthinkable only ten years ago. Being able to solve the DCR problem optimally for several categories of schedulers allows us to compare these categories as for QoS routing performance, i.e., to better characterize the cost that has to be payed, in terms of network utilization, for using lower-complexity schedulers. This is particularly relevant in view of a relatively new fact that has recently modified the landscape of QoS routing, i.e., that centralized path computation is now being advocated in several networking architectures - e.g., those based on Path Computation Elements [20, the control plane of Software Defined Networks [21, industrial networks, avionic networks, and cyber-physical systems - where explicit guarantees on the delivery time are to be 105 coupled with the resource usage efficiency that only an entity with a global knowledge of the network status can provide. Hence, centralized, optimal path computation solution is nowadays a viable approach, allowing us to employ techniques that would have been unsuitable under the 
assumption that path computation was meant to be done by routers themselves, hence had to be simple and rely on local state information only. This means that theoretical network performance improvements related to the use of more efficient scheduling algorithms may in fact translate into practice in several applications environments.

The rest of the paper is organized as follows: Section 2 reviews the related work. Section 3 introduces the system model, and Section 4 states the problem formally and outlines our solution approaches. These are evaluated numerically in Section 5 Finally, Section 6 reports conclusions and highlights directions for future work on this topic.

\section{Related Work}

We now describe the most relevant related work on QoS routing and optimal path computation. QoS routing is concerned with finding paths subjects to QoS constraint, such as a minimum guaranteed bandwidth, a maximum delay or jitter, etc.. A seminal paper on the topic is [22], which shows that computing a shortest path under two or more additive or multiplicative constraints is $\mathcal{N} \mathcal{P}$-complete. For instance, per-link delays are additive, and per-link loss probabilities are multiplicative. However, path computation with one additive/multiplicative constraint and concave constraints (such as a minimum available bandwdith along a path) is instead polynomial.

There has been a large amount of literature devoted to finding approximate solutions for the work offline (the so-called "pre-computation" approach) to make the online part faster 27. All the above works, however, consider link delay as a static per-link metric, hence neglect queueing. Relatively fewer works 13, 14, 28, aim to find paths and per-link rate reservations that meet a pre-specified non-additive end-to-end worst-case delay. This is possible because the WCD is a the flow is upper bounded [29]. The above works assume PGPS schedulers [1] and leaky-bucketshaped flows, and endeavour to find a path with enough available rate. It has been shown in [28] that path computation under the above assumptions is a polynomial problem if the rates to be reserved at each link are the same and known in advance. Later, [13] and [14 have shown that be different, instead, makes the problem $\mathcal{N} \mathcal{P}$-hard. However, our previous works [15, 16, have shown that constraining the rates to be the same at all links comes with a high cost in terms of network performance: removing that assumption, in fact, abates the flow rejection probability considerably. Moreover, even though path computation becomes non-polynomial, it is still solvable rates is practicable. Furthermore, heuristic approaches can be devised that are much faster than exact solution methods while achieving solution of similar quality, thus reinforcing the above claim. 
All these works only consider PGPS schedulers. However, their results can obviously be applied without any modification to every scheduler whose latency expression is the same as PGPS, i.e.,

145 on the impact that these expressions have on optimization problems - can be found in 30. In that work, group-based approximations of WFQ are not mentioned, since they have only appeared more recently. To the best of our knowledge, this is the first work considering delay-constrained routing using non-SRP schedulers. As we show later on, employing non-SRP scheduler is not

straightforward from a modeling point of view, since it introduces admission control constraints, on one hand, and requires some clever modeling choices, on the other. We terminate this section by observing that there are indeed many works comparing schedulers, along the dimensions of latency, fairness and complexity. While almost every work proposing a new scheduler somewhat positions it in the above three-dimensional space, some works [2, 3] show that the three axes are not independent, and what fundamental trade-offs bound them together. To the best of our knowledge, no work so far compared schedulers regarding their suitability to QoS routing.

\section{Background and system model}

This section details the hypotheses underlying our contribution, and provides the necessary background on worst-case delay computation and latency expressions.

The network where flows have to be routed is represented as a directed graph $G=(N, A)$, where $N$ is the set of nodes (i.e., routers or switches) and $A$ is the set of $\operatorname{arcs}$ (i.e., links) joining them. A node $i \in N$ is characterized by a fixed node delay $n_{i}$, representing the time it takes for a packet to travel from an input interface to an output interface. A link $(i, j) \in A$ is characterized by its constant propagation delay $l_{i j}$ and its capacity $w_{i j}$. Moreover, the maximum transmit unit (MTU) $L$ is known and assumed to be constant throughout the network for simplicity.

We focus on a tagged flow to be routed through the network, and call $s \in N$ and $d \in N \backslash\{s\}$ its source and destination nodes. The flow has an end-to-end deadline $\delta$, and the routing must be such that the WCD of the flow must not exceed $\delta$. A WCD guarantee can only be given if the injection rate of the flow is upper-bounded. Such upper bound can be expressed in the form of an arrival curve $A(\tau): \mathbb{R}_{+} \rightarrow \mathbb{R}_{+}$, which is a wide-sense increasing function bounding from above the number of bits that the flow is allowed to send in any interval of length $\tau$. That is, if $F(t)$ measures the overall number of bits injected by the flow by time $t$, we have $F(t+\tau)-F(t) \leq A(\tau)$ for all $t$ and $\tau \geq 0$. We assume that the arrival curve is affine, i.e. $A(\tau)=\sigma+\rho \cdot \tau$. This curve is often referred to in the literature as the leaky-bucket arrival curve, and its two non-negative parameters $\sigma$ and $\rho$ are called burst and rate, respectively.

We assume that each link is managed by a WFQ scheduler (e.g., PGPS, DRR, etc.), where each flow traversing the link has to specify its reserved rate for the link. Obviously, in order to 
verify whether or not a feasible routing exists for the new flow it is necessary to know the current state of the network. This is specified by the set $K$ of existing flows: each flow in $k \in K$ is characterized by its chosen path $p(k)$ between its source and its destination, its deadline $\delta^{k}$, its burst and rate parameters $\sigma^{k}$ and $\rho^{k}$, and its reserved rates $r_{i j}^{k}$ for each link $(i, j) \in p(k)$. We will similarly denote by $p$ the path chosen for the new rate, and by $r_{i j}$ the chosen reserved rates for $(i, j) \in p$. Finally, we will denote by $P(i, j)=\{k \in K:(i, j) \in p(k)\}$ the set of existing flows traversing the arc $(i, j)$; note that this does not comprise the new one, which has yet to be routed. All this data is clearly required to define the conditions under which a feasible path $p$ and reserved rates exist for the new flow. For instance, any WFQ scheduler guarantees that - over a suitable interval of time - the new flow will be served at least with the required rate $r_{i j}$, regardless of the presence of other flows; however, this holds only provided that the link is not oversubscribed, i.e.,

$$
r_{i j}+\sum_{k \in P(i, j)} r_{i j}^{k} \leq w_{i j}
$$

Also, in order for the WCD to be finite the minimum rate among all links of the path must be at least as large as the traffic injection rate of the flow, i.e.,

$$
r_{i j} \geq \rho \quad \forall(i, j) \in p
$$

Under (2), the WCD along path $p$ is

$$
\frac{\sigma}{\min \left\{r_{i j}:(i, j) \in p\right\}}+\sum_{(i, j) \in p}\left(\theta_{i j}+l_{i j}+n_{i}\right)
$$

where $\theta_{i j}$ is the latency that the flow experiences on link $(i, j)$. That latency models the delay that the head-of-line packet of the tagged flow undergoes due to the scheduling process, and its expression varies from one scheduler to the other. Following and extending [30, we can classify WFQ schedulers into four classes, depending on their latency expressions:

- Strictly Rate-Proportional (SRP) latency, i.e., the one of Packet-by-packet GPS (PGPS, 1], also called WFQ) and Worst-case Fair Weighted Fair Queueing $\left(\mathrm{WF}^{2} \mathrm{Q}\right.$, [31]). The expression for SRP latency is:

$$
\theta_{i j}=\frac{L}{w_{i j}}+\frac{L}{r_{i j}}
$$

Note that the latency is inversely proportional to the reserved rate, barring an additive constant $L / w_{i j}$ (which is unavoidable and due to atomic packet transmission), hence the name SRP. Thus, the latency can be reduced by increasing the flow's reserved rate. It has been proved that SRP latency can only be achieved at $O(\log n)$ worst-case per-packet complexity [2, 3], $n$ being the number of flows traversing the link.

- Group-Based (GB) approximations of $W F Q$, e.g. [8, 9]. In these schedulers, flows are grouped by requested bandwidth at logarithmic intervals, which ensures $O(1)$ complexity at the price 
of a larger latency. The link latency expression is 8

$$
\theta_{i j}=2 \frac{L}{w_{i j}}+3 \frac{2^{\left\lceil\log _{2} w_{i j} L / r_{i j}\right\rceil}}{w_{i j}},
$$

which can be easily shown to satisfy

$$
2 \frac{L}{w_{i j}}+3 \frac{L}{r_{i j}} \leq \theta_{i j} \leq 2 \frac{L}{w_{i j}}+6 \frac{L}{r_{i j}} .
$$

Hence, the latency is still rate-proportional, but with a constant $(\geq 3)$ multiplying the ratedependent term and a larger constant offset: thus, it is in general larger than (4). We call (5) a Group-Based latency.

- Schedulers with Weakly Rate-Proportional (WRP) latency, e.g. Self-clocked Fair Queueing [10. SCFQ was introduced as a simpler approximation of the GPS paradigm, since it does not need to emulate a GPS scheduler. However, it still exhibits logarithmic complexity, and its latency depends on the number of flows $|P(i, j)|$ traversing the link simultaneously:

$$
\theta_{i j}=|P(i, j)| \frac{L}{w_{i j}}+\frac{L}{r_{i j}} .
$$

If $|P(i, j)|$ is large, increasing the reserved rate may decrease the latency only marginally, hence the name given to this class.

- Frame-Based (FB) schedulers, such as DRR [4] and similar [5, 6, which achieve $O(1)$ complexity by imposing that flows are visited in a fixed order, each for a minimum amount of time (called a quantum). The quantum determines the guaranteed rate, which is in fact the ratio of the quantum to the round duration. Thus, in these schedulers latency depends on the number of flows, but also on their quantum, hence on the reserved rate for each flow. Note that $O(1)$ complexity can only be guaranteed if all quanta are lower bounded. In DRR, the quantum lower bound is equal to the MTU $L$. Moreover, quantum allocation also reflects rate partitioning, i.e., one flow is guaranteed double the rate as another flow if and only if its quantum is twice as large. This implies that the flow requesting the minimum reserved rate must get a quantum equal to the lower bound $L$, and all other flows get their quantum accordingly. Thus, the latency expression of DRR, besides the number of flows, also depends on the reserved rates of other flows on the link. More specifically, it depends on both their sum

$$
\bar{r}_{i j}=\sum_{k \in P(i, j)} r_{i j}^{k}
$$

and their minimum

$$
r_{i j}^{m i n}=\min \left\{r_{i j}^{k}: k \in P(i, j)\right\}
$$


The latency of the DRR scheduler has been computed in [6]; it is easy to verify that the formula obtained therein can be rewritten as

$$
\theta_{i j}=\frac{L}{w_{i j}} \frac{\bar{r}_{i j}}{\min \left\{r_{i j}, r_{i j}^{\min }\right\}}+|P(i, j)| \frac{L}{w_{i j}}+\frac{L}{r_{i j}} .
$$

Other frame-based schedulers (e.g. [5, 6]), which are variants of the basic DRR scheme, achieve smaller latencies by scaling down the lower bound on the quanta (i.e., below the MTU size) by a constant factor $\kappa$, and avoiding the complexity penalty by using clever data structures. The resulting latency has the similar expression

$$
\theta_{i j}=\frac{L}{w_{i j}} \frac{\bar{r}_{i j}}{\kappa \cdot \min \left\{r_{i j}, r_{i j}^{m i n}\right\}}+|P(i, j)| \frac{L}{w_{i j}}+\frac{L}{r_{i j}} .
$$

The limit for $\kappa \rightarrow \infty$ exactly reproduces the WRP latency (7). However, the cost of the scheduling algorithm, either in time or in space if implemented in hardware, grows with $\kappa$, which means that $\kappa$ cannot be taken arbitrarily large. Yet, clever implementations allow to select $\kappa$ so as to get quite close to the WRP latency, at a reasonable cost; the interested reader is referred to [6] for details. For simplicity, in the following we will only work with $\kappa=1$, as employing a generic $\kappa>0$ would not change the shape of the models.

The aim of this paper is to formulate and solve the Delay-constrained Routing (DCR) problem: given the current state of the network and a set of link reservation costs $f_{i j}>0$-i.e., the cost of reserving one unit of capacity on $(i, j)$ - find one feasible $s$ - $d$ path, and a feasible reserved rate at each of its links, so that the flow can be routed along the path and meet its end-to-end deadline at the minimum possible reservation cost. Obviously, admitting a new flow must not jeopardize the delay guarantees of other flows already present in the network. This is a practical concern, since both the latency formulas of WRP (7) and FB (8) schedulers include terms that depend on the number of flows traversing the link. Furthermore, (8) also includes the minimum rate reserved for a flow at that link. Therefore, admitting a new flow, by increasing some other flow's latency, might make them violate their deadline, in which case the new flow must be rejected. In other words, the admission of a new flow, for these schedulers, requires global admission control to ensure that all other established flows keep meeting their deadlines. Note that this problem cannot occur with SRP or GSRP schedulers, since their latencies only depend on the rate of the new flow being routed. For these, therefore, global admission control is not required, and the admission of a new flow is only conditioned by the availability of rate along the chosen path. So far, the DCR problem has only been dealt with in the context of SRP schedulers in [15, 16], where it has been proven to be a Mixed-Integer Second Order Cone problem (MI-SOCP). In the following, we formulate it for the other categories of schedulers - notably, those requiring global admission control. 


\section{Mathematical programming formulation}

In [15, 16], the DCR problem for SRP schedulers was formulated as a MI-SOCP problem. We recall that formulation hereafter, as a baseline to understanding its generalizations.

To model routing, we use binary variables $x_{i j} \in\{0,1\}$ to indicate whether link $(i, j)$ belongs to $p$ : this allows us to write down the standard flow conservation constraints

$$
\sum_{(j, i) \in B S(i)} x_{j i}-\sum_{(i, j) \in F S(i)} x_{i j}=\left\{\begin{array}{rl}
-1 & \text { if } i=s \\
1 & \text { if } i=d \\
0 & \text { otherwise }
\end{array} \quad i \in N .\right.
$$

If the (indirect) cost of setting any $x_{i j}=1$ is positive, 9 ensure that - at optimality - the $x$ variables represent an $s$ - $d$ path. In (9), $B S(i)$ is the subset of $A$ containing the arcs entering node $i$ (the so-called "backward star" of the node), while $F S(i)$ is the subset of $A$ containing the arcs leaving node $i$ (the so-called "forward star" of the node). We then introduce rate reservation variables $r_{i j}$, that are instead continuous, and an additional variable $r_{m i n}$, with an obvious meaning. For these we define the reservable capacity $c_{i j} \leq w_{i j}-\bar{r}_{i j}$ at each arc: $r_{i j} \leq c_{i j}$ ensures that (1) holds. Note that $c_{i j}$ may be chosen to be strictly smaller than $w_{i j}-\bar{r}_{i j}$ if some of the link capacity has to be kept for other uses (signaling, backup paths, ...). Then, the constraints

$$
\begin{array}{ll}
0 \leq r_{i j} \leq c_{i j} x_{i j} & (i, j) \in A \\
\rho \leq r_{\text {min }} \leq r_{i j}+c_{\max }\left(1-x_{i j}\right) & (i, j) \in A
\end{array}
$$

ensure on one hand that $r_{i j}=0$ if $x_{i j}=0$, and on the other hand that $\rho \leq r_{\min } \leq r_{i j} \leq c_{i j}$ if $x_{i j}=1$, so that both (1) and (2) hold. Note that $c_{\max }=\max \left\{c_{i j}:(i, j) \in A\right\}$ is used in (11) to ensure that any link not in the chosen path $\left(x_{i j}=0\right)$ does not contribute to bounding $r_{\text {min }}$ from above.

The constraint on the WCD given by (3) can be modeled using an auxiliary variable $t$ and a rotated $S O C P$ constraint as follows:

$$
\begin{aligned}
& t+\sum_{(i, j) \in A}\left(\theta_{i j}+\left(l_{i j}+n_{i}\right) x_{i j}\right) \leq \delta \\
& t r_{\text {min }} \geq \sigma \quad, \quad t \geq 0
\end{aligned}
$$

Note that the $l_{i j}$ and $n_{i}$ terms in the sum in 12 are only counted in if $x_{i, j}=1$, i.e., if the link and node are actually in the chosen path $p$. For the same reason, some care must be taken to constrain the latency variable $\theta_{i j}$ to be equal to zero if $x_{i j}=0$, or to an appropriate (convex) nonlinear expression otherwise, which in the SRP case looks as follows:

$$
\theta_{i j}=\left\{\begin{array}{ll}
\frac{L}{r_{i j}}+\frac{L}{w_{i j}} & \text { if } x_{i j}=1 \\
0 & \text { if } x_{i j}=0
\end{array} .\right.
$$


This is a disjunctive set, being expressed by a disjunction, which is in general nonconvex. Also note that $x_{i j}=0 \Longrightarrow r_{i j}=0$, which renders the $L / r_{i j}$ term ill-defined. In [15] it is proven that the best approach to represent a disjunctive set makes use of Perspective Reformulation techniques 32, 18, 33, and results in:

$$
\begin{array}{ll}
\theta_{i j}=L s_{i j}+\left(L / w_{i j}\right) x_{i j} & (i, j) \in A \\
s_{i j} r_{i j} \geq x_{i j}^{2}, \quad s_{i j} \geq 0 & (i, j) \in A
\end{array}
$$

Clearly, the " $\left(L / w_{i j}\right) x_{i j}$ " term in (14 can be merged into the corresponding " $\left(l_{i j}+n_{i}\right) x_{i j}$ " term in 12 , obtaining

$$
t+\sum_{(i, j) \in A}\left[L s_{i j}+\left(L / w_{i j}+l_{i j}+n_{i}\right) x_{i j}\right] \leq \delta
$$

subject to 15. Finally, we assume the linear objective function

$$
\min \sum_{(i, j) \in A} f_{i j} r_{i j}
$$

i.e., we minimize the weighted amount of allocated rate along the path using the given reservation $\operatorname{costs} f_{i j}$. The whole model is thus a Mixed-Integer Second Order Cone Program, due to the (rotated) conic constraints (13) and (15). MI-SOCPs can be solved (although, in general, not in polynomial time) by off-the-shelf, efficient, general-purpose solvers like Cplex or GUROBI. We remark that different formulations of some of these constraints are possible, which may result in the solvers to be able to solve the problems somewhat more efficiently in some cases, as discussed in 34. However, the presented formulation is already efficient enough for all the cases we test, hence we stick to it for simplicity. We also remark that formulation (9-17) only requires knowledge of the other flows in the definition of the reservable capacities $c_{i j}$. In fact, with SRP schedulers, once a set of feasible rate reservations has been computed so that the new flow meets the required deadline, there is no need to check whether the other, already admitted flows still meet theirs provided that no link is over-reserved, i.e., (1) holds. Therefore, no further admission control constraints are required. This is no longer true for the other schedulers, which therefore require a different analysis.

\subsection{Generalizations to other latency models}

We now show that similar MI-SOCP models can also be derived when GB, WRP and FB schedulers are used. We refer the readers to [34] for more details on the derivations and possible alternative models.

\subsubsection{Group-based Schedulers}

The embodiment of (3) to the case of GB schedulers, whose latency is (5), is not straightforward. In fact, (5) is non-smooth, given the ceiling operator on the exponent. This would lead to complex 
non-linear, non-convex models, for which very few solvers are available and that could hardly be solved to optimality in split seconds. However, it is easy to observe that both the lower and upper bounds to (5) shown in (6) lead to convex models. In particular, using the upper bound in (6) - which is a safe choice, since we are discussing worst-case performance - we can just replace (14) with

$$
\theta_{i j}=6 L s_{i j}+\left(2 L / w_{i j}\right) x_{i j}
$$

leaving (15) unchanged; this means that 16 becomes

$$
t+\sum_{(i, j) \in A}\left[6 L s_{i j}+\left(2 L / w_{i j}+l_{i j}+n_{i}\right) x_{i j}\right] \leq \delta .
$$

Because the latter only differs from 16 in two coefficients, there is no impact on the shape of the optimization model. Also, no "explicit" admission control mechanism is required in this case, save checking that enough capacity is available for the new flow similarly to the SRP case.

\subsubsection{Weakly Rate-Proportional Schedulers}

We now consider the latency model (7), which includes term $|P(i, j)|$. Again, the embodiment of (3) in this case is straightforward: just replace (14) with

$$
\theta_{i j}=L s_{i j}+\left(L / w_{i j}\right)|P(i, j)| x_{i j}
$$

leaving (15) unchanged. As $|P(i, j)|$ does not depend on the rates, this again has no impact on the shape of the optimization model. Note, however, that in this case admission control is required. In fact, unlike with SRP and GB, in this case a flow's latency depends on $|P(i, j)|$, which changes (for some arcs) if a new flow is admitted. Hence, we need to ensure that existing flows still meet their deadline after the new flow is admitted; if this is not the case, the new flow must be rejected, even though there may be enough rate for it to meet its own deadline. For each active flow $k \in K$, we therefore define the delay slack $\bar{\delta}^{k}$

$$
\bar{\delta}^{k}=\delta^{k}-\frac{\sigma^{k}}{\min \left\{r_{i j}^{k}:(i, j) \in p(k)\right\}}-\sum_{(i, j) \in p(k)}\left(\frac{L}{r_{i j}^{k}}+(|P(i, j)|-1) \frac{L}{w_{i j}}+l_{i j}+n_{i}\right)
$$

which represents the amount of extra delay that flow $k$ can tolerate, without changing either its path $p(k)$ or its reserved rates $r_{i j}^{k}$, while still meeting its deadline $\delta^{k}$. Note that the term $|P(i, j)|-1$ comes from the fact that the term " $|P(i, j)|$ " in (7) does not count $k$, as it refers to the status where $k$ had not been routed yet. On the contrary, when defining $\bar{\delta}^{k}$ the flow has already been routed, and it is obviously true that $k \in P(i, j)$ for all $(i, j) \in p(k)$ (which in particular means that $|P(i, j)|-1 \geq 0)$. Now, in order to ensure that the delay of $k$ along $p(k)$, using the fixed reserved rates $r_{i j}^{k}$, does not increase more than $\bar{\delta}^{k}$, it is sufficient to add the linear admission control constraint

$$
\sum_{(i, j) \in p(k)}\left(L / w_{i j}\right) x_{i j} \leq \bar{\delta}^{k}
$$


It goes without saying that the model is still MI-SOCP, since the above admission control constraints are linear. Of course, one needs one constraint 20 for each $k \in K$, and therefore the number of added constraints w.r.t. the original SRP and GB formulation is $O(|K|)$.

\subsubsection{Frame-based Schedulers}

Finally, we consider FB schedulers. When comparing their latency expression against WRP's, (4), it is apparent that FB latency includes both a "simple" additive term $\left(L / w_{i j}\right)|P(i, j)|$ like (7), and the rate-dependent term

$$
\frac{L}{w_{i j}} \frac{w_{i j}-r_{i j}}{\min \left\{r_{i j}, r_{i j}^{m i n}\right\}}
$$

However, clearly, 21) only applies if $x_{i j}=1$, i.e., arc $(i, j)$ is chosen to be in the path for the new flow; in fact, the term would otherwise go to $+\infty$ when $x_{i j}=0 \Longrightarrow r_{i j}=0$. Now, 21] is not a jointly convex function in $r_{i j}$ and all the $r_{i j}^{k}\left(r_{i j}^{m i n}\right)$. However the latter are fixed in this setting and therefore so is $r_{i j}^{m i n}$; this makes 21 convex in $r_{i j}$. In fact, the function

$$
\phi\left(r_{i j}\right)=\left(w_{i j}-r_{i j}\right) / \min \left\{r_{i j}, r_{i j}^{\min }\right\}
$$

that describes (up to a constant) 21] can be rewritten as

$$
\phi\left(r_{i j}\right)=\left\{\begin{array}{ll}
\phi_{1}\left(r_{i j}\right)=w_{i j} / r_{i j}-1 & \text { if } r_{i j} \leq r_{i j}^{\min } \\
\phi_{2}\left(r_{i j}\right)=\left(w_{i j}-r_{i j}\right) / r_{i j}^{\text {min }} & \text { if } r_{i j} \geq r_{i j}^{\text {min }}
\end{array} .\right.
$$

It is then immediate to see geometrically, and easy to verify algebraically, that not only $\phi_{1}\left(r_{i j}^{\text {min }}\right)=$ $\phi_{2}\left(r_{i j}^{m i n}\right)$, but also $\phi_{1}\left(w_{i j}\right)=\phi_{2}\left(w_{i j}\right)[=0]$. It follows that $\phi_{2}\left(r_{i j}\right) \geq \phi_{1}\left(r_{i j}\right)$ for all $r_{i j} \in\left[r_{i j}^{m i n}, w_{i j}\right]$, whereas $\phi_{1}\left(r_{i j}\right) \geq \phi_{2}\left(r_{i j}\right)$ for $r_{i j} \in\left(0, r_{i j}^{m i n}\right]$. Hence, in the interval $\left[\rho, w_{i j}\right]$ that matters for our problem one can alternatively define

$$
\phi\left(r_{i j}\right)=\max \left\{\phi_{1}\left(r_{i j}\right), \phi_{2}\left(r_{i j}\right)\right\}
$$

Hence, using standard representation of convex max-functions we can formulate 21] as

$$
\begin{array}{ll}
\theta_{i j}=L s_{i j} \frac{L}{w_{i j}}|P(i, j)| x_{i j}+v_{i j} & (i, j) \in A \\
v_{i j} \geq L s_{i j}-L / w_{i j}, v_{i j} \geq\left(L / r_{i j}^{\text {min }}\right) x_{i j}-L r_{i j} /\left(w_{i j} r_{i j}^{\text {min }}\right), v_{i j} \geq 0 & (i, j) \in A
\end{array}
$$

(again, including (15) as well).

As can be expected, admission control constraints for FB schedulers are more complex than WRP's due to the need to express the nonlinear term 211. However, one can use the same definition of delay slack used for WRP; this is because, as already mentioned, the FB latency is equal to WRP's plus the rate-dependent addendum. As the latter depends on the choices made for 
the new flow, hence is not constant, it cannot be included in the delay slack, i.e., the righ-hand-side of the constraint. In other words, one can write the admission control constraint as

$$
\sum_{(i, j) \in p(k)} \frac{L}{w_{i j}}\left(x_{i j}+\frac{w_{i j}-r_{i j}^{k}}{\min \left\{r_{i j}, r_{i j}^{m i n}\right\}}\right) \leq \bar{\delta}^{k}
$$

with $\bar{\delta}^{k}$ of 19 . This is clearly related to the WRP version 20, but for the extra part 21]; it has to be remarked, again, that that term only applies when $x_{i j}=1 \Longrightarrow r_{i j}>0$ (otherwise it would send that term to $+\infty$ ). Exploiting the already discussed properties of 21], a SOCP formulation can be easily obtained:

$$
\begin{array}{ll}
\sum_{(i, j) \in p(k)}\left(L / w_{i j}\right)\left(x_{i j}+\left(w_{i j}-r_{i j}^{k}\right) z_{i j}\right) \leq \bar{\delta}^{k} & \\
z_{i j} \geq 1 / r_{i j}^{m i n}, \quad z_{i j} \geq s_{i j} & (i, j) \in p(k)
\end{array}
$$

Note that the $1 / r_{i j}^{m i n}$ term is always well-defined because $(i, j) \in p(k)$, hence arc $(i, j)$ is not

empty - it contains at least the flow $k$-and therefore $r_{i j}^{m i n}>0$. It is also important to remark that neither the variables $s_{i j}$ nor the $z_{i j}$ depend on the flow $k$; that is, these can be defined just once for all $\operatorname{arcs}(i, j) \in A$ and then used to define the admission constraints for all the active flows. Actually, the $z_{i j}$ variables only need to be defined for all $(i, j)$ for which at least one active flow is routed. Therefore, in this case as well the model is still a MI-SOCP, despite the additional admission control constraints. Note that their number is higher than those of WRP, as constraints (23) are $O(|K|)$, whereas $(24)$ are $O(|K|+|A|)$; also, some $O(|A|)$ extra variables are needed.

\section{Numerical Results}

We now analyze the impact on network performance of employing different schedulers for QoS routing. In general, simpler schedulers come with higher latencies, and this must reflect on their ability to admit traffic in the network. We analyze this effect by measuring the blocking probability, i.e., the relative ratio of unfeasible path computations, in several scenarios. We also show that solving to optimality the DCR problem is affordable with all the scheduler classes: the solution time is invariably well below one second, on off-the-shelf hardware, even for large networks and for a wide range of loads. This has already been shown for SRP schedulers in [16]; we now extend that result by showing that even factoring in admission control constraints (for WRP and FB schedulers) does not increase the computational burden significantly. Furthermore, modeling improvements may further improve the efficiency of the solution process, as discussed in [34.

\subsection{Simulation setup}

Constructing a set of meaningful instances to compare the various scheduling classes is a nontrivial exercise. We follow the guidelines of our earlier paper [16], which we summarize here for 
ease of reading. A number of real-world IP network topologies, shown in Table1, are taken from the Internet Topology Zoo [35]. These topologies are heterogeneous with respect to network dimension, connectedness (represented by the average node rank) and geographic span (summarized by the average per-link propagation delay), ranging from regional (e.g., Belnet2009) to world-wide (e.g., DeutscheTelekom). Link delays $l_{i j}$ are set according to the geographic coordinates, dividing the geodesic distance between $i$ and $j$ by the speed of light in a fiber. Node delays are selected equal to $40 \mu \mathrm{s}$, a figure that can be expected to be on the safe side according to [36]. Link capacities are assigned using the FNSS tool [37, and selected among $\{1,10,40\}$ Gbps, according to the edge betweenness centrality metric. FNSS also generates realistic traffic matrices based on the network capacity, and we exploit this to generate the $\rho$ value of each request so that it can be accepted in an unloaded network. Traffic matrices are generated using a log-normal distribution with a mean rate equal to $0.8 \mathrm{Gbps}$ and a variance of 0.05 . The MTU $L$ is fixed to 1500 bytes. Flow deadlines $\delta$ are set with the following process. We first compute two extreme values: $\delta_{\min }$, corresponding to the minimum feasible deadline obtained by allocating the entire link capacity and then calculating the delay-shortest path, using SRP schedulers, given this fixed allocation, and $\delta_{\max }$, corresponding to the delay bound obtained by allocating a rate equal to $\rho$ on all the links of the shortest path, still using SRP schedulers. Delay requests smaller than $\delta_{\min }$ cannot be met, whereas requests higher than $\delta_{\max }$ are likely to make the delay constraint redundant with SRP schedulers. Thereafter, $\delta$ is randomly chosen uniformly within the interval $\left[\delta_{\min }, \delta_{\min }+\beta\left(\delta_{\max }-\delta_{\min }\right)\right]$ for a fixed parameter $\beta \in(0,1)$; the smaller $\beta$, the more difficult meeting the delay constraint can be expected to be.

Path computation request inter-arrivals are exponential with a varying rate $\lambda$ : each path lasts for an exponentially distributed time with a mean equal to $1 \mathrm{~s}$, hence $\lambda$ represents the number of erlangs. The number of path computations requested is large enough to estimate blocking probabilities correctly even at low values of $\lambda$. Each point in the graphs is obtained as the average of five independent replicas, and 95\% confidence intervals are also reported.

Simulations have been performed on a 2.299 Ghz AMD Opteron 6376 with 16Gb RAM, running a 64 bits Linux operating system (Ubuntu 12.4). All the codes were compiled with gcc 4.4 .3 and -03 optimizations. The MI-SOCPs were solved by off-the-shelf commercial solver Cplex 12.6, ran with default parameters. Thus, it is not unlikely that just tweaking the algorithmic parameters of the solver could buy us an additional speedup.

\subsection{Results: blocking probability}

We simulate path computation with all the scheduler classes, namely SRP, WRP, FB and GB; for the latter we use the lower bound approximation of the latency given in (5), for reasons that will become clear soon. We plot the blocking probability in Figure 1, as a function of $\lambda$, for all the above topologies when $\beta=0.2$ and $\sigma=3 \mathrm{MTU}$. 

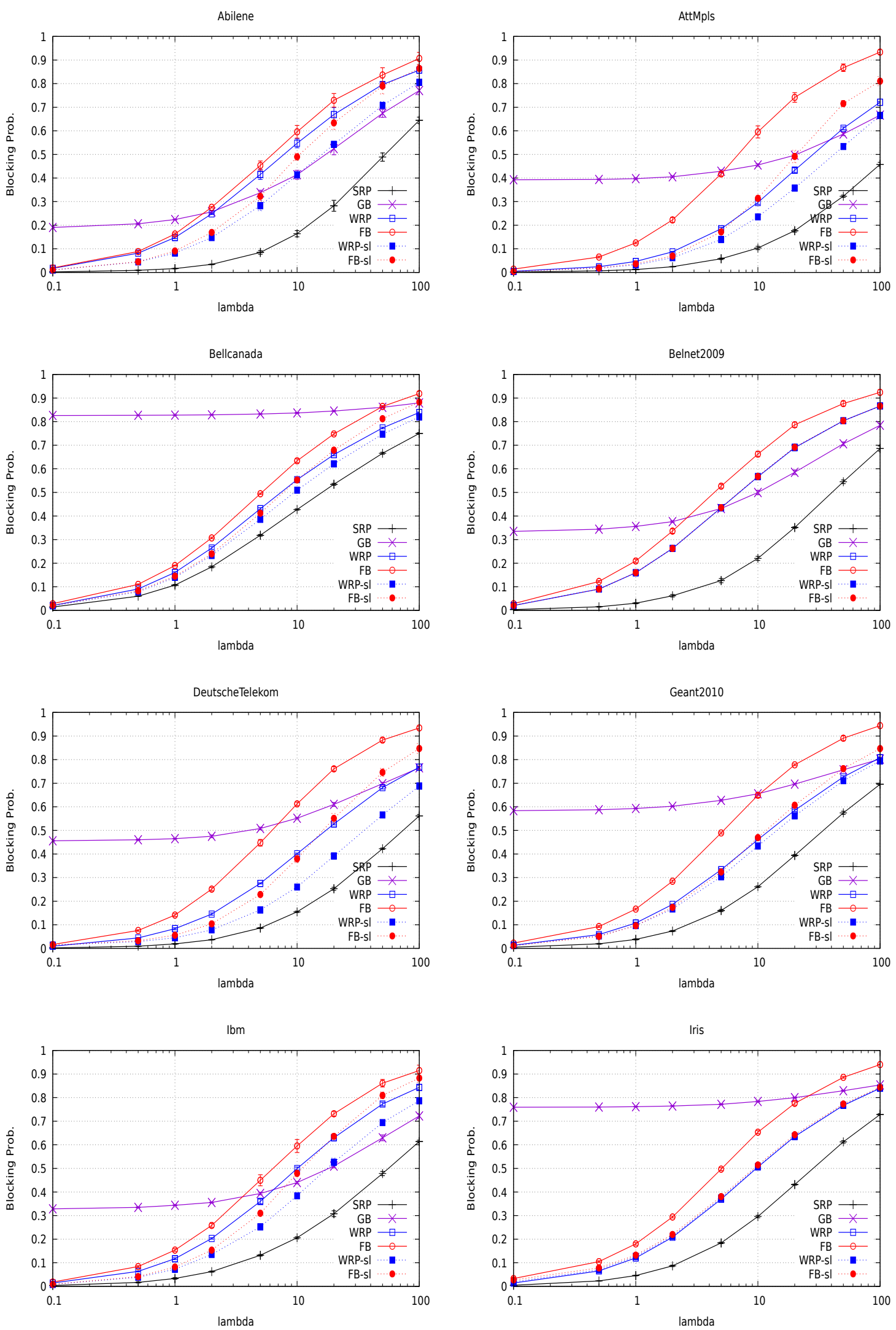

Figure 1: Blocking probability for all topologies, $\beta=0.2$ and $\sigma=3 \mathrm{MTU}$ 
Table 1: Topologies used in the simulations (Topology Zoo dataset, 35]).

\begin{tabular}{|l|r|r|r|r|r|}
\hline Topology & \# nodes & \# links & \# flows & avg. node rank & avg. prop. delay (ms) \\
\hline Abilene & 11 & 28 & 110 & 2.55 & 5.03 \\
AttMpls & 25 & 112 & 600 & 4.48 & 4.54 \\
Bellcanada & 48 & 128 & 2256 & 2.67 & 2.83 \\
Belnet2009 & 21 & 48 & 420 & 2.29 & 0.19 \\
DeutscheTelekom & 39 & 124 & 912 & 3.18 & 13.79 \\
Geant2010 & 37 & 112 & 1332 & 3.03 & 3.93 \\
Ibm & 18 & 48 & 306 & 2.67 & 4.67 \\
Iris & 51 & 128 & 2550 & 2.51 & 0.27 \\
\hline
\end{tabular}

For all the schedulers classes, the blocking probability ranges from negligible (when $\lambda=0.1$ ) to almost certain (when $\lambda=100$ ). The figure clearly shows that SRP outperforms all the other scheduler classes. What makes GB perform poorly is the fact that latency (5) includes a factor two to multiply the rate-dependent term. This fact is unique among all scheduler classes, and is such that GB is often unable to meet the deadlines (which, we recall, were already challenging for SRP schedulers) even in an unloaded network. Note that we have chosen a lower bound approximation on purpose, so as to discount the hypotheses that GB's poor performance were due to a pessimistic upper-bound approximation.

The charts show that WRP and FB perform considerably worse than SRP, although they exhibit the same dynamics; that is, their blocking probability does decrease at small loads, unlike that of GB. Among them, FB performs considerably worse than WRP, which is expected in that it always has larger latency for the same allocated rates (cf. the discussion about the extra term (21) in 4.1.3. The performance gap between WRP and SRP could be explained similarly, at least for larger loads where several active flows are present on the network: cf. the discussion about the extra term $|P(i, j)|$ in the latency formulæ of 4.1 .2 . However, a deeper analysis shows that an entirely different factor is also at play here: namely, admission control. The relevant mechanism is the following: when a flow is admitted, its rates are computed based on the current value of $|P(i, j)|$ (and of $r_{\min }$ for $\mathrm{FB}$ ). In particular, because a minimum-cost allocation is sought, the smallest possible rates are computed. Given the inverse proportionality between rates and delay, this means that, for the given path, the selected rates are those that produce the largest possible feasible delay, i.e., $\delta$. In other words: when a flow is admitted, its delay slack 197 is necessarily null. The consequence of this choice is that, unless some flow disappears later on, any other flow that attempts to use the same links will increase $|P(i, j)|$, thus increasing the WCD of any existing flow: but because the WCD is already at its maximum, even a fractional improvement is impossible. Thus, a new flow may be found to be impossible to route not because there is not 
enough rate to support it, but because of the fact that it would disrupt the current flows (i.e., because of admission control). In other words: once any flow seizes a link $(i, j)$, it prevents any other flow from using it until it is removed.

The only way to get around this problem is to overallocate rates somewhat, under WRP and FB, so as to buy flows some slack at their admission. This can, for instance, be done by decreasing the deadline by a small percentage in the WCD constraint, so as to force the model to allocate more rates than these that would actually be necessary. That is, we can substitue the deadline constraint (12) with

$$
t+\sum_{(i, j) \in A}\left(\theta_{i j}+\left(l_{i j}+n_{i}\right) x_{i j}\right) \leq \delta(1-\varepsilon)
$$

for some small $\varepsilon>0$. This way, some delay slack is introduced to protect the flow from a subsequent increase in $|P(i, j)|$ and/or decrease in $r_{\min }$. Of course, one should not overdo it, lest the problem is made unfeasible because the deadline is made too strict, or the increase in rate consumption makes it impossible for other flows to be admitted for lack of available capacity that could have been admitted otherwise. In order to verify that this mechanism is indeed significant we have repeated the experiments, for WRP and $\mathrm{FB}$, with the small value $\varepsilon=5 \cdot 10^{-5}$. The results are again plotted in Figure 1 (lines "WRP-sl" and "FB-sl"), alongside the non-slackened approaches. The charts show that even a small slack is enough to abate the blocking probability substantially in most topologies, thus confirming that the admission control is a significant factor for the blocking probability of WRP and SF schedulers.

\subsection{Results: running times}

Average computation times for all schedulers are shown in Figure 2 proving that computation times, being in the tens to hundreds of milliseconds, are indeed affordable. As observed in [16], computation times depend on the topology, and specifically on both its connectedness and dimension, the former having larger impact on the size of the solution space than the latter. All schedulers exhibit a decreasing trend with the load: this is because the optimization problem becomes unfeasible at higher loads, as testified by the increase in the blocking probability, and the solver detects unfeasible problems faster than it solves feasible ones of the same size. This phenomenon also explains why SRP is not consistently the fastest one despite having the simplest model; indeed, especially for large loads SRP usually requires more time than the other schedulers, largely because it has a lower failure rate. For analogous reasons, the "slackened" variants (WRP-sl and FB-sl) usually require more time than the corresponding non-slackened one (WRP and FB). On the contrary, at lower loads, where the failure rate of all the schedulers is comparable, the other models often are (fractionally) more costly to solve than SRP, which is expected due to them being more complex. In fact, FB is also typically (fractionally) more costly to solve than WRP, since it has more complex constraints, both for describing the latency of the new flow and 

do not generate major overhead in the solution time, as testified by the fact that WRP and FB computation times, both with and without slack, are comparable to those of SRP and GB.

\section{Conclusions and future research}

In this paper we have proposed - to the best of our knowledge, for the first time - a centralized path computation and resource allocation approach that can be employed with all classes of fairqueueing schedulers. We believe that our work provides the following interesting contributions:

- It is possible to formulate the DCR problem for all relevant classes of fair-queueing schedulers as MI-SOCPs; this was not a foregone conclusion, since there was no a-priori guarantee that latency formulæ had to be convex, and a fortiori Second-Order Cone representable.

- As a consequence, there is an actual possibility to use WRP and FB schedulers for QoS routing purposes, using a centralized-decision approach, employing standard off-the-shelf optimization tools. The overhead of doing this is comparable to that of using more complex SRP schedulers, which again was not obvious since the corresponding mathematical models are more complex.

- However, WRP and FB perform worse than SRP in terms of network performance, i.e., gains in implementation complexity have to be payed - possibly, dearly - for in terms of blocking probability. While this could be expected, since WRP and FB have, all the rest being equal, higher latency than SRP, the magnitude of the difference really is relevant. This means that when designing a network for sustaining highly delay-sensitive flows, the choice of the scheduling protocol at the routers may play a more important role than is currently realized. In particular GB schedulers, while being a clever $O(1)$ approximation of SRP ones, perform rather poorly as far as QoS routing is concerned, even if we solve the problem at optimality while using a lower-bound approximation for the latency, thereby requiring smaller rates than what would actually be needed to meet the required QoS bound. To the best of our knowledge, our results are the first that offer insight in the trade-off between the cost of the scheduling protocol and the quality of the corresponding QoS routing.

- Our results for the first time reveal that the policy of minimizing the sum of rates allocated to the newly routed flow, which has hitherto been assumed in all the works on QoS scheduling, may not be the best one when admission control is required. In other words, minimizing the rate, while intuitively appealing, is not necessarily a good proxy for actual network performances, as measured e.g. by blocking probability. This means that different notions of "quality of obtained path and rates" will have to be developed in order to be embodied in the 

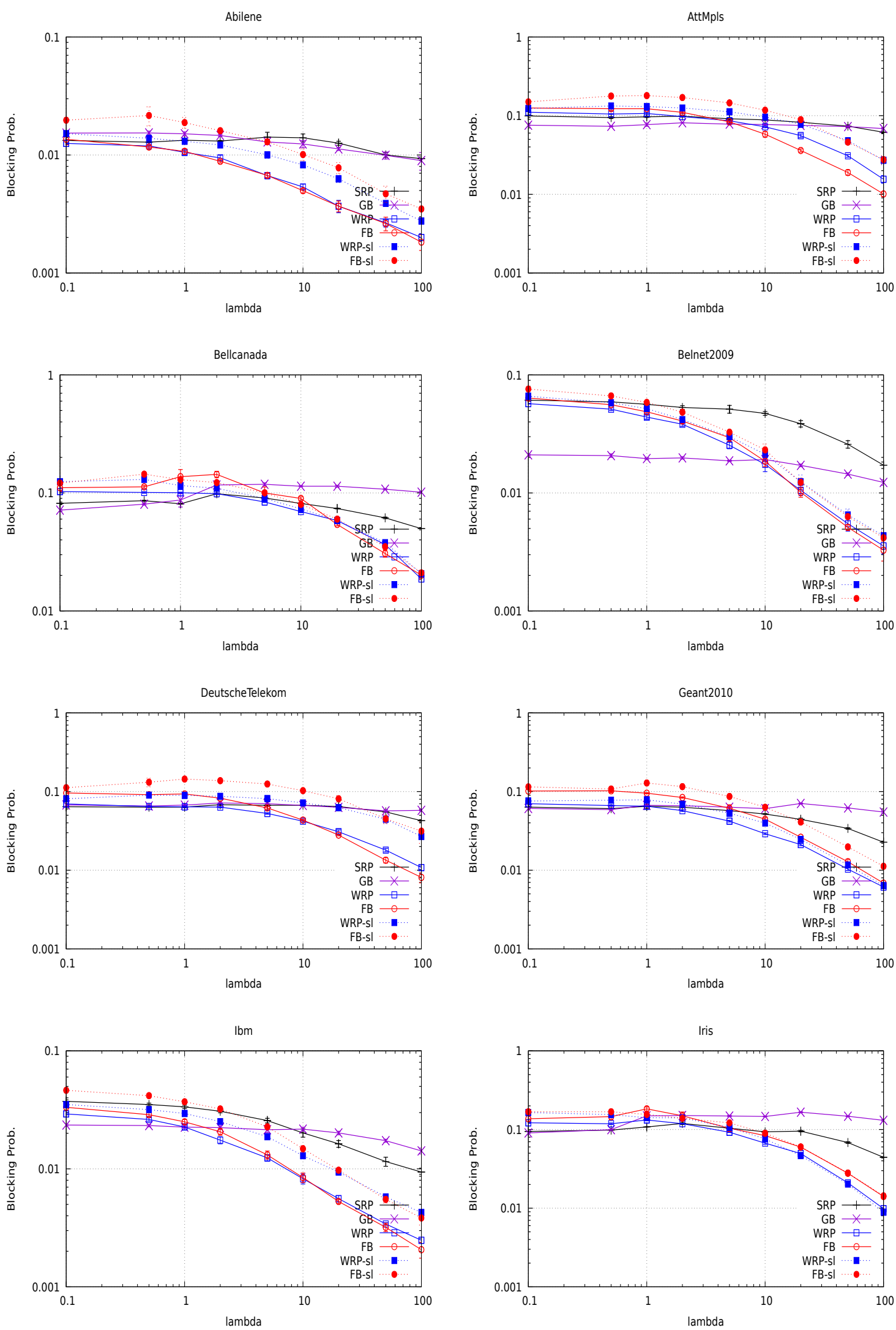

Figure 2: Average computation times for all topologies, $\beta=0.2$ and $\sigma=3 \mathrm{MTU}$ 
mathematical models. We have proposed a first, simple approach to this issue by selecting a fixed delay slack, but it is apparent that our proposal is a somewhat crude one: there must be an optimal slack - since both a null and a "high" ones lead to poor performance-but estimating it does not seem to be trivial.

We therefore believe that the topic deserves future investigations. In particular, it seems to be important to come up with more appropriate objective functions and/or constraints describing the quality of QoS routing than just the sum of the used rates along the links. As an example, it would be interesting to investigate slack selection policies for schedulers requiring admission control.

\section{Acknowledgements}

The first and second authors gratefully acknowledge the contribution of the project of the University of Pisa "Mathematical models and computational methods for complex networks". The second author has been funded by the FIRB 2013 project RBFR13ZYQL of the Italian Science and Education Ministry. This research has also been partly undertaken under the auspices of the PRIN 2012 project 2012JXB3YF "Mixed-Integer Nonlinear Optimization: Approaches and Applications" of the Italian Science and Education Ministry.

[1] K. Parekh, R. Gallager, A Generalized Processor Sharing Approach to Flow Control in Integrated Services Networks: the Single Node Case, IEEE/ACM Trans. on Networking 1 (1993) 344-357.

[2] J. Xu, R. Lipton, On fundamental tradeoffs between delay bounds and computational complexity in packet scheduling algorithms, in: SIGCOMM, 2002, pp. 279-292.

[3] P. Valente, Exact gps simulation with logarithmic complexity, and its application to an optimally fair scheduler, in: SIGCOMM, 2004, pp. 269-280.

[4] M. Shreedhar, G. Varghese, Efficient fair queueing using deficit round robin, IEEE/ACM Transactions on Networking 4 (1996) 375-385.

[5] L. Lenzini, E. Mingozzi, G. Stea, Eligibility-Based Round Robin for Fair and Efficient Packet Scheduling in Interconnection Networks, IEEE Transactions on Parallel and Distributed Systems 15 (3) (2004) 254-266.

[6] L. Lenzini, E. Mingozzi, G. Stea, Tradeoffs between low complexity, low latency and fairness with deficit round robin schedulers, IEEE/ACM Transactions on Networking 12 (4) (2004) 681-693. 
[7] L. Lenzini, E. Mingozzi, G. Stea, Performance Analysis of Modified Deficit Round Robin Schedulers, IOS Journal of High-Speed Networks 16 (4) (2007) 399-422.

[8] F. Checconi, L. Rizzo, P. Valente, QFQ: Efficient packet scheduling with tight guarantees, IEEE/ACM Trans. Netw. 21 (3) (2013) 802-816.

[9] M. Karsten, Approximation of generalized processor sharing with interleaved stratified timer wheels, IEEE/ACM Trans. Netw. 18 (3) (2010) 708-721.

[10] S. Golestani, A Self-Clocked Fair Queueing Scheme for Broadband Applications, Proc. of IEEE INFOCOM'94, Toronto, Canada (1994) 636-646.

[11] D. Lorenz, A. Orda, Optimal partition of QoS requirements on unicast paths and multicast trees, IEEE/ACM Transactions on Networking 10 (2002) 102-114.

[12] M. Saad, A. Leon-Garcia, W. Yu, Optimal Network Rate Allocation under End-to-End Quality-of-Service Requirements, IEEE Transactions on Network and Service Management 4 (3) (2007) 40-49.

[13] Q. Ma, P. Steenkiste, Quality-of-Service Routing for Traffic with Performance Guarantees, in: In Proc. IFIP International Workshop on Quality of Service, 1997, pp. 115-126.

[14] A. Orda, Routing with End-to-End QoS Guarantees in Broadband Networks, IEEE/ACM Trans. on Networking 7 (3) (1999) 365-374.

[15] A. Frangioni, L. Galli, M. Scutellà, Delay-constrained shortest paths: Approximation algorithms and second-order cone models, Journal of Optimization Theory and Applications 164(3).

[16] A. Frangioni, L. Galli, G. Stea, Optimal joint path computation and rate allocation for realtime traffic, The Computer Journal Vol. 58 No. 6.

[17] S. Burer, A. N. Letchford, Non-convex mixed-integer nonlinear programming: A survey,

口 Surveys in Operations Research and Management Science 17 (2) (2012) 97 - 106. doi:http: //dx.doi.org/10.1016/j.sorms.2012.08.001. URL http://www.sciencedirect.com/science/article/pii/S1876735412000037

[18] A. Frangioni, C. Gentile, Perspective Cuts for a Class of Convex 0-1 Mixed Integer Programs, Mathematical Programming 106 (2) (2006) 225-236.

[19] A. Frangioni, C. Gentile, A Computational Comparison of Reformulations of the Perspective Relaxation: SOCP vs. Cutting Planes, Operations Research Letters 37 (3) (2009) 206-210. 
[20] F. Paolucci, F. Cugini, A. Giorgetti, N. Sambo, P. Castoldi, A Survey on the Path Computation Element (PCE) Architecture, IEEE Communications Surveys Tutorials.

[21] Open Networking Foundation, Software-Defined Networking: The New Norm for Networks, White paper, Open Networking Foundation, Palo Alto, CA, USA (Apr. 2012). URL http://www.opennetworking.org/images/stories/downloads/sdn-resources/ white-papers/wp-sdn-newnorm.pdf

[22] Z. Wang, J. Crowcroft, Qos routing for supporting resource reservation, IEEE Journal on Selected Areas in Communications.

[23] T. Korkmaz, M. Krunz, Multi-constrained optimal path selection, in: Proceedings of INFOCOM'01, 2001.

[24] X. Yuan, Heuristic Algorithms for Multiconstrained QoS routing, ACM/IEEE Trans. on Networking.

[25] F. Kuipers, T. Korkmaz, M. Kruntz, An overview of constraint-based path selection algorithms for QoS routing, IEEE Communications Magazine.

[26] A. Juttner, B. Szviatovszki, I. Mecs, Z. Rajko, Lagrange relaxation based method for the QoS routing problem, in: Proceedings of INFOCOM'01, 22-26 April 2001, Anchorage, Alaska USA, IEEE, 2001, pp. 859-868.

[27] A. Orda, A. Sprinston, QoS routing: the precomputation perspective, in: Proceedings of INFOCOM'00, 26-30 March 2000, Tel Aviv, Israel, IEEE, 2000, pp. 128-136.

[28] C. Pornavalai, G. Chakraborty, N. Shiratori, QoS based routing algorithm in integrated services packet networks, Proceedings of ICNP'97, 1997, pp. 167-174.

[29] J.-Y. L. Boudec, P. Thiran, Network Calculus: A Theory of Deterministic Queuing Systems for the Internet, Springer LNCS, 2001.

[30] A. Lori, G. Stea, G. Vaglini, Towards Resource-Optimal Routing Plans for Real-Time Traffic, in: T. Margaria, B. Steffen (Eds.), Leveraging Applications of Formal Methods, Verification, and Validation, Vol. 6415 of Lecture Notes in Computer Science, 2010, pp. 214-227.

[31] J. Bennett, H. Zhang, WF2Q: Worst-case fair weighted fair queueing, in: INFOCOM, 1996, pp. $120-128$.

[32] A. Frangioni, F. Furini, C. Gentile, Approximated Perspective Reformulations: a Project\&Lift Approach, Mathematical Programming (submitted). 
[33] A. Frangioni, C. Gentile, E. Grande, A. Pacifici, Projected Perspective Reformulations with Applications in Design Problems, Operations Research 59 (5) (2010) 1225-1232.

[34] A. Frangioni, L. Galli, G. Stea, Delay-constrained routing problems: Accurate scheduling models and admission control, Technical Report, Dipartimento di Informatica, Università di Pisa (2015).

[35] The internet topology zoo.

490 URL http://www .topology-zoo.org/

[36] K. Papagiannaki, S. Moon, C. Fraleigh, P. Thiran, C. Diot, Measurement and analysis of single-hop delay on an ip backbone network, IEEE Journal on Selected Areas in Communications 21(6) (2003) 908-921.

[37] L. Saino, C. Cocora, G. Pavlou, A toolchain for simplifying network simulation setup, in: 495 Proceedings of the 6th International ICST Conference on Simulation Tools and Techniques, SIMUTOOLS '13, ICST, ICST, Brussels, Belgium, Belgium, 2013. 